\title{
Anmerkung zu Fredrik Roggan: Am deutschen Rechtswesen soll die Welt genesen?
}

Die nach dem Urteil des BGH zur Verbreitung der Auschwitz-Lüge im Internet ${ }^{1}$ geäußerte polemische Kritik geht fehl. Zum einen ist eine Anwendbarkeit des deutschen Strafrechts für das potentielle Gefährdungsdelikt der Volksverhetzung ${ }^{2}$ auch dann gegeben, wenn ein Ausländer im Ausland den Holocaust leugnet, zum anderen liegt hierfür auch ein völkerrechtlich legitimierender Anknüpfungspunkt durch den Bezug der »Auschwitz-Lüge« auf das Gebiet der Bundesrepublik Deutschland vor. Es ist weitgehend anerkannt, daß eine bloße Wahrnehmbarkeit im Inland der im Ausland begangenen Tathandlung beim abstrakten Gefährdungsdelikt keinen Inlandstatort nach $\$ 9$ I I. Alt. StGB (die Tat ist an dem Ort begangen, an dem der Täter gehandelt hat) begründet; ${ }^{3}$ auch wenn es dem Täter gerade auf eine Wahrnehmung im Inland ankommt: Er handelt im Ausland. ${ }^{4}$ Daher kann bei diesen Delikten eine Geltung des deutschen Strafrechts nur dann begründet werden, wenn der in $\$ 9$ I 3. Alt. StGB vorausgesetzte "zum Tatbestand gehörende Erfolg " in Deutschland eintritt. Dem wird entgegen gehalten, daß abstrakte Gefährdungsdelikte keine Erfolgsdelikte sind und somit auch keinen "zum Tatbestand gehörenden Erfolg « i.S. d. $\$ 9$ StGB haben könnten.'

Der »zum Tatbestand gehörende Erfolg « wird im Allgemeinen Teil des Strafgesetzbuchs jedoch nicht in dem Sinne verstanden, daß zwar konkrete, nicht aber abstrakte Gefährdungsdelikte von dieser Umschreibung erfaßt werden. So wird ein "zum Tatbestand gehörender Erfolg « für die Strafbarkeit des Unterlassens einer Handlung ( $\$ 13$ StGB) vorausgesetzt, ohne daß bezweifelt wird, daß auch abstrakte Gefährdungsdelikte durch ein Unterlassen verwirklicht werden können; ${ }^{6}$ ebenso wird eine Anwendbarkeit der Verjährungsregeln nach $\ 78$ a S. 2 StGB für möglich gehalten.? Eine Beschränkung des $\$ 9$ I 3. Alt. StGB auf Erfolgsdelikte läßt sich dem Gesetzeswortlaut also nicht entnehmen. Vielmehr ist für den Erfolgsbegriff des $\$ 9$ StGB nicht auf Veränderung der Außenwelt durch eine Handlung, sondern auf eine tatbestandliche Beeinträchtigung des geschützten Rechtsguts abzustellen. ${ }^{8}$

Zudem ist weiterhin fraglich, worin genau der in diesem Zusammenhang postulierte Unterschied zwischen abstrakten und konkreten Gefährdungsdelikten in Hinblick auf einen Handlungserfolg im Sinne einer Veränderung der Außenwelt zu suchen ist. Denn bei konkreten Gefährdungen wird lediglich eine Handlung mit Strafe bedroht, die die hohe Wahrscheinlichkeit des Eintritts einer bestimmten Folge begründet, ohne daß es zum Eintritt dieser Folge kommen muß, ' während bei abstrakten Gefähr-

\footnotetext{
I BGH, Urt. v. I 2. I 2.2000-I StR I 84/00, NJW 200I, S. 624 ff: Ein australischer Staatsbürger hatte auf einem australischen Server Inhalte ins Internet eingespeist, in denen der Holocaust geleugnet wurde.

2 Als Unterfall eines abstrakten Gefährdungsdelikts, vgl. Lackner/Kühl, 23. Aufl. München r 999, Rn. 32 vor $\$$ I 3; Tröndle/Fischer, 50.Aufl. München 2001, Rn. I 3a vor $\$ 13$.

3 Anders bei der Wahrnehmbarkeit über eine Fernsehausstrahlung im Inland: KG Berlin, NJW r999, S. 3500 $3502 ; \mathrm{vgl}$. auch krit. Anmerkung von Heinrich, NStZ $2000, \mathrm{~S} .533 \mathrm{ff}$.

4 Wobei sich der Vorsatz ohnehin nicht auf den Tatort erstrecken muß, vgl. Schönke/Schröder-Eser, 26. Aufl. München 2001, \$9 Rn. I s, Lackner/Kühl, $\$ 9$ Rn. I.

5 Schönke/Schröder-Eser, $\$ 9$ Rn. 6; Hilgendorf, NJW 1997, 1875.

$6 \mathrm{Vgl}$. umfassend Heinrich, GA I999, S. $72 \mathrm{ff}$.

7 BGHSt 36, 255, 257.

8 Vgl. Heinrich, GA 1999, S. 79

9 Dazu Roxin, 3. Aufl München 1997, \$10 Rn. x23.
} 
dungen eine angenommene generelle Gefährlichkeit der Handlung genügt. ${ }^{10} \mathrm{Da}$ es also bei beiden Gefährdungsarten nicht auf einen Erfolg ankommt, wäre auch schon die Anwendbarkeit des $\$ 9$ I 3. Alt. StGB auf konkrete Gefährdungsdelikte problematisch. ${ }^{\text {II }}$

Der BGH hat in seiner Entscheidung ausdrücklich offen gelassen, ob abstrakte Gefährdungsdelikte unter $\$_{9} I_{3}$. Alt. StGB fallen können, da er sich bei der Volksverhetzung auf ein potentielles Gefährdungsdelikt stützen konnte und die im Tatbestand der Volksverhetzung geforderte (konkrete) Eignung zur Friedensstörung im Inland als Anknüpfungspunkt für das deutsche Strafrecht nutzte. Jedoch bleibt darüber hinaus festzustellen, daß aus den oben genannten Gesichtspunkten eine Anwendbarkeit deutschen Rechts über $\$ 9$ I 3 . Alt. StGB auch bei abstrakten Gefährdungsdelikten grundsätzlich möglich ist. Dabei kann der Wille des Täters, eine Wirkung im Inland zu erzielen, nicht als Ausschlußkriterium herhalten: Da die Merkmale des $\$ 9 \mathrm{StGB}$ nicht zum Unrechtstatbestand gehören, muß sich der Vorsatz nicht auf diese erstrecken. ${ }^{12}$

Die grundsätzliche Anwendbarkeit deutschen Strafrechts auch auf abstrakte Gefährdungsdelikte, bei denen der Täter im Ausland handelt, bedarf allerdings des einschränkenden Korrektivs eines völkerrechtlich legitimierenden Anknüpfungspunktes. ${ }^{13}$

Für die in $\$ 130$ III StGB ausdrücklich normierte Leugnung der Völkermorde unter der Herrschaft der Nationalsozialisten besteht dieser - wie der BGH zu Recht feststellt - durch den besonderen Bezug dieser Taten zu Deutschland..$^{14}$ Wann allerdings ein solcher Anknüpfungspunkt generell besteht, hat der BGH in seiner Entscheidung offen gelassen, und hierin ist der eigentliche Kern des Problems zu sehen. Ein Ausschluß der abstrakt gefährlichen Delikte schon aus dem Anwendungsbereich des $\$ 9 \mathrm{I}$ 3. Alt. StGB ist nur ein die eigentliche Problemstellung vorwegnehmender Versuch, die Reichweite des Strafrechts einzudämmen. Begrenzend ist ein objektiver Bezug der Tat zur Bundesrepublik zu verlangen, der sich im vorliegenden Fall aus der geschichtlichen Verantwortung der Deutschen für die Völkermorde unter der Herrschaft der Nationalsozialisten ergab.

Der Befürchtung, deutsche Strafverfolgungsorgane würden nunmehr global als wWeltpolizei« agieren und abweichend von den Prinzipien des Völkerrechts eine de facto Zensur des Meinungsaustausches im Internet betreiben, ist übertrieben. Es ist zwar nicht von der Hand zu weisen, daß der sehr weite Tatbestand der Volksverhetzung ${ }^{15}$ als "Einstiegsnorm « für Ermittlungen gegen Rechtsextremisten ${ }^{16}$ eine erhebliche Bedeutung hat, die sich mit der Neufassung des G-10 Gesetzes bedenklich erweitern wird ${ }^{17}$, doch stehen die tatsächlichen Möglichkeiten zu mehr als einer fragmentarischen Kontrolle der Inhalte des Internets im Widerspruch zur Ermittlungsökonomie der Strafverfolgungsorgane; die Ermittlung eines Tatverdächtigen, dessen Auslieferung nach und letztlich Anklage in Deutschland erscheint zweifelhaft ${ }^{18}$.

Io Wobei mit absoluter Ungefährlichkeit der Handlung sogar eine abstrakte Gefährdung im Einzelfall entallen kann, vgl. BGHSt 26, 12 r.

i Vgl. auch Sieber, NJW 1999, S. 2069, 2070.

12 In diesem Sinne auch Hilgendorf, NJW 1997, S: 1876.

13 Dazu Werle/Jeßberger, JuS $2001, \mathrm{~S} .35 \mathrm{ff}$.

14 Siehe Clauß, MMR 2001, S. 232, 233.

15 Zur Entwicklung des Tatbestandes: Frommel, KJ I 995 , S. $402 \mathrm{ff}$

16 Erstaunlich in diesem Zusammenhang sind die seit Jahren auffallend niedrigen Fallzahlen zu $\$ 130 \mathrm{StGB}$ in Sachsen-Anhalt im Vergleich zu allen anderen Bundesländern.

17 Gleichstellung der Volksverherzung mit der Bildung terroristischer Vereinigungen ( $\$ 129 \mathrm{a}$ StGB), vgl. BTDs. $14 / 5655$; BR-Ds. $351 / 01$.

$18 \mathrm{In}$ dem vom BGH entschiedenen Fall wurde der Täter allerdings in Deutschland verhaftet. 